\title{
有机分子聚集体中振动分辨光谱的激子耦合效应
}

\author{
李文强 $^{a} \quad$ 彭谦 $^{*}, b \quad$ 谢育俊 $^{c} \quad$ 张天 $^{a} \quad$ 帅志刚*, \\ $\left({ }^{a}\right.$ 清华大学化学系 北京 100084) \\ ${ }^{b}$ 中国科学院化学研究所 北京 100190) \\ ( ${ }^{c}$ 武汉大学化学系 武汉 430072)
}

\begin{abstract}
摘要 光谱是探究分子间相互作用及发光机理的有效手段. 本工作采用 Frenkel 激子模型和量子力学/分子力学 $(\mathrm{QM} / \mathrm{MM})$ 方法系统研究了一系列聚集诱导发光(AIE)体系和传统荧光(非 AIE)体系晶态下的吸收、发射光谱. 结果表明, 分子内电声子耦合 $(\lambda)$ 与分子间激子耦合 $(J)$ 竞争决定了晶态聚集体的光谱特性. 在室温下，当 $J / \lambda$ 值大于约 0.17 时，有机 分子聚集体光谱的激子耦合效应将表现明显. 例如, 对于面对面排列的 $\mathrm{H}$ 聚集体，只有考虑激子耦合效应的理论计算 光谱才与其实验光谱吻合很好, 即相较于单分子光谱的吸收蓝移、发射减弱并红移. 对于 AIE 体系, 因为其 $J / \lambda$ 值均小 于 0.17 , AIE 聚集体光谱特征主要是由分子内电声子耦合所主导，激子耦合可以忽略不计. 关键词 有机分子聚集体; Frenkel 激子模型; 电声子耦合; 激子耦合效应; QM/MM 方法
\end{abstract}

\section{Effect of Intermolecular Excited-state Interaction on Vibrationally Resolved Optical Spectra in Organic Molecular Aggregates}

\author{
Li, Wenqiang $^{a} \quad$ Peng, Qian*, ${ }^{*}$ Xie, Yujun ${ }^{c} \quad{\text { Zhang, } \text { Tian }^{a} \quad \text { Shuai, Zhigang }}^{*, a}$ \\ ( ${ }^{a}$ Department of Chemistry, Tsinghua University, Beijing 100084) \\ $\left({ }^{b}\right.$ Institute of Chemistry, Chinese Academy of Sciences, Beijing 100190) \\ ( ${ }^{c}$ Department of Chemistry, Wuhan University, Wuhan 430072)
}

\begin{abstract}
The optical spectra are effective means to reveal the molecular interactions and the luminescent mechanism of the organic molecules in aggregates. Herein, we systematically investigate the crystalline state vibrationally resolved absorption and emission spectra for a series of AIEgens and non-AIEgens by considering intermolecular excited state interaction by using Frenkel-exciton model coupled with quantum mechanics and molecular mechanics $(\mathrm{QM} / \mathrm{MM})$ calculations. It is found that the competition between the intramolecular vibronic coupling $(\lambda)$ and the intermolecular exciton coupling $(J)$ governs the crystalline aggregate spectral characters. At room temperature, when $J / \lambda$ value is larger than a critical value (ca. 0.17), the exciton coupling would have a large effect on the optical spectra. For face-to-face H-aggregates, only when both intermolecular electrostatic and excitonic couplings are considered, can one obtain calculated vibrationally resolved spectra and well reproduce the experimental results, namely, remarkable blue-shift in absorption but much less red-shift in emission when compared with the gas-phase. The optical spectra of the AIE-active aggregates are determined by the intramolecular vibronic coupling because the ratio $J / \lambda$ is less than the critical value.

Keywords organic molecular aggregate; Frenkel exciton model; intramolecular vibronic coupling; intermolecular exciton coupling; QM/MM
\end{abstract}

\section{Introduction}

Organic materials have been a research hotspot owing to their potential applications in promising optoelectronic devices, such as organic light emitting diodes, ${ }^{[1]}$ lasing, ${ }^{[2]}$ field-effect transistors, ${ }^{[3]}$ sensors, ${ }^{[4]}$ solar cells, ${ }^{[5]}$ and so on. The solid-state photophysics is different from solution owing to a variety of intermolecular interactions. The measurable optical spectrum is an effective means to reveal the molecularly oriented packing feature and the luminescent mechanism of organic molecules in aggregates. For instance, comparing with solution-phase optical spectra, the rod-shaped molecules always show a blue shift in absorption but red shift and quenching in emission for typical $\mathrm{H}$-aggregates while their absorption and emission both are red-shifted and the emission intensity is noticeably enhanced in J-aggregates. Differently, for the typical aggre-

\footnotetext{
*E-mail: qpeng@iccas.ac.cn, Tel.: 010-82616830; zgshuai@tsinghua.edu.cn, Tel.: 010-62797689

Received August 30, 2016; published November 3, 2016.

Supporting information for this article is available free of charge via the Internet at http://sioc-journal.cn

Project supported by the Ministry of Science and Technology of China through the 973 program (Grants 2013CB834703, 2015CB65502 and 2013CB933503), the National Natural Science Foundation of China (Grants 21473214, 21290191 and 91233105), and the Strategic Priority Research Program of the Chinese Academy of Sciences (Grant XDB12020200).

项目受科技部 973 计划(013CB834703, 2015CB65502,2013CB933503), 国家自然科学基金(21473214, 21290191, 91233105)及中国科学院战略性先导科 技专项(XDB12020200)资助.
} 
gation induced emission (AIE) compounds, when going from solution to aggregate, the absorption peak is almost unchanged but emission is blue-shifted. ${ }^{[6]}$ The fine structure of the vibrationally resolved optical spectrum can provide much information of molecular geometrical modification between the ground and excited states, electronic transition property, intermolecular interaction and resonance energy transfer (intermolecular excited-state interaction or exciton coupling) by comparing the solid-phase spectrum with the gas-phase or solution-phase ones. ${ }^{\text {[] }}$ Therefore, it is imperial to describe the vibrationally resolved spectrum by considering both the intramolecular and intermolecular interaction as well as the exciton coupling from first principles, and figure out the origin of the spectral peaks, which helps to deeply understand the mechanism of luminescence and to identify important molecular parameters governing the luminescent property of organic molecules in aggregates.

Kasha $^{[8]}$ has sketched out the spectral feature for $\mathrm{H} / \mathrm{J}$-aggregates by taking resonance energy transfer process into consideration on the basis of a simple model from a purely electronic state view. For H-aggregates, the absorption peak goes blue shift while the fluorescence is quenched when compared with those in solution. For J-aggregates, both absorption and emission are red-shifted and swelled in intensity. Recently, Spano et al. ${ }^{[9]}$ revealed the relationship between the intensity of 0-0 transition and $\mathrm{H}$ or J-aggregate conformation. Gierschner et al. ${ }^{[10]}$ found the intermolecular Herzberg-Teller vibronic transitions by calculating the excited state property of the cluster consisting of several molecules at time-dependent density functional theory (TDDFT) level. Liang et al. ${ }^{[11]}$ attributed the large red-shifted emission of PTCDI to the intermolecular charge transfer character. For flexible AIE systems, Wu et al. ${ }^{[12]}$ claimed that the spectral character with unchanged absorption and blue-shifted emission upon crystallization are mainly caused by the intramolecular vibronic coupling and intermolecular electrostatic interaction at the level of quantum mechanics and molecular mechanics (QM/MM) theory.

In this contribution, we unveil the effect of exciton coupling on the vibrationally resolved solid-state optical spectral for organic molecules aggregates in crystalline state for the first time considering the intramolecular vibronic coupling, intermolecular excitonic coupling, and the intermolecular electrostatic interaction. Based on the Frenkel exciton model, we analyze the competitive relationship between the intramolecular vibronic coupling and intermolecular exciton coupling. And then we quantitatively calculate the optical spectra of a series of crystalline systems ranging from conventional non-AIEgens to novel AIEgens and reveal the mechanism of different spectrum nature in the non-AIEgens and AIEgens' aggregates, which can provide a clear relationship between structure and optical property.

\section{Result and Discussion}

\subsection{Exciton model of molecular spectroscopy}

\subsubsection{Electronic-state exciton model}

Taking a dimer as an example, the basis function of the Frenkel exciton without considering vibration states can be written as $|e, 1\rangle=|e(1), g(2)\rangle$ and $|e, 2\rangle=|g(1), e(2)\rangle$, and the model Hamiltonians reads

$$
\boldsymbol{H}_{e}=\left(\begin{array}{ll}
\varepsilon & J \\
J & \varepsilon
\end{array}\right)
$$

Here $J$ is the exciton coupling; $\varepsilon$ is the molecular excitation energy. By diagonalizing the matrix, we can get the eigenvalue and eigenvector,

$$
E^{ \pm}=\varepsilon \pm|J|, \quad \psi_{ \pm}=\frac{\sqrt{2}}{2}\left(|e, 1\rangle \pm \frac{J}{|J|}|e, 2\rangle\right)
$$

The constructed eigenstates are called as the Frenkel exciton (FE) states. The dependence of optically spectral feature on $J$ is numerously examined by setting $\varepsilon=3.0 \mathrm{eV}$ at $298 \mathrm{~K}$ in Figure 1. For the $\mathrm{H}$-aggregates, the transition dipole moment is arranged parallels and the resultant exciton coupling $J$ is positive, so the lowest FE state is a dipole-forbidden transition and the fluorescence is quenched according to Kasha rule. ${ }^{[13]}$ The absorption processes appear in higher dipole-allowed FE states and exhibit remarkable blue shift. While, for the J-aggregates, the

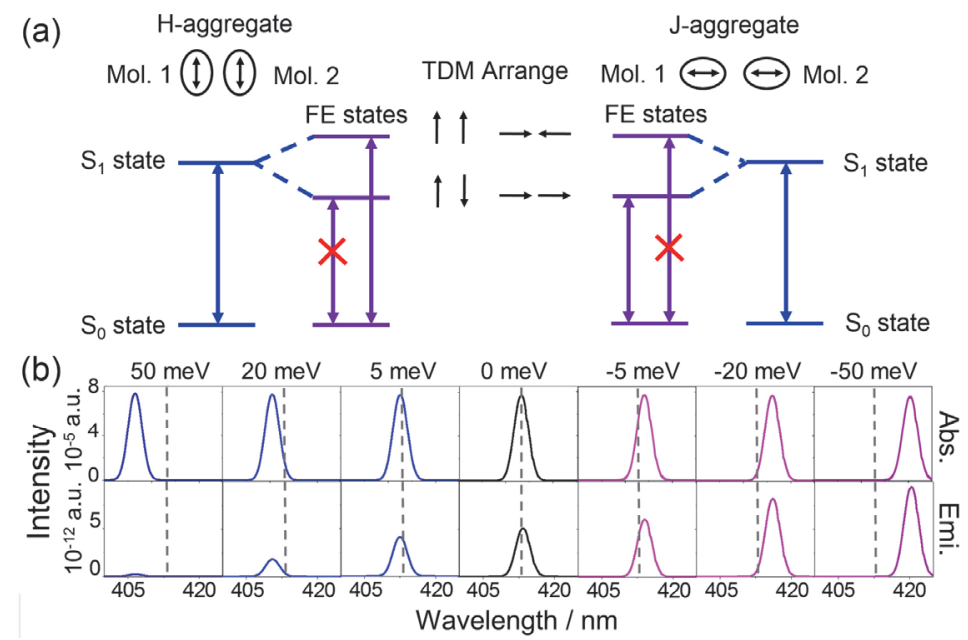

Figure 1 Exciton band energy diagram (a) and the dependence of the optical spectra on the exciton coupling in the H- and J-aggregates at $298 \mathrm{~K}$ (b). TDM: transition dipole moment 
$J$ is negative, resulting in the dipole-allowed transition of the lower FE state. Consequently, and the absorption and emission are both red-shifted and the emission intensity is sharply enhanced. It is also seen that the shifts of the spectra become very serious when the $J>20 \mathrm{meV}$.

\subsubsection{Vibronic-coupled exciton model}

As known, the vibronic coupling is essential in determining the spectral shape ${ }^{[14]}$ For simplification, a dimer system with one normal mode with two quantum number $(v=0$ and 1) for each molecule is considered under the vibronic coupled exciton model. The model exciton Hamiltonian is constructed as

$$
\boldsymbol{H}_{e}=\left(\begin{array}{cccc}
1 / 2 \hbar \omega+\varepsilon & 0 & J \xi_{0-0} \xi_{0-0} & J \xi_{1-0} \xi_{0-0} \\
0 & 3 / 2 \hbar \omega+\varepsilon & J \xi_{0-0} \xi_{0-1} & J \xi_{1-0} \xi_{0-1} \\
J \xi_{0-0} \xi_{0-0} & J \xi_{0-0} \xi_{0-1} & 1 / 2 \hbar \omega+\varepsilon & 0 \\
J \xi_{1-0} \xi_{0-0} & J \xi_{1-0} \xi_{0-1} & 0 & 3 / 2 \hbar \omega+\varepsilon
\end{array}\right)
$$

Here, the $\xi_{0-v}\left(\xi_{0-v}=\xi_{v-0}\right)$ is the Franck-Condon integration between the ground state of quantum number $v=0$ and the excited state of $v$ and can be calculated by using $\xi_{0-v}^{2}=\frac{S^{v}}{v !} e^{-s}, S=\frac{\omega}{2 \hbar} D^{2}$, in which $S$ represents the Huang-Rhys factor and $D$ is the displacement between the equilibrium geometries of the ground and excited states. By diagonalizing the Hamiltonian (seen in Supporting Information, SI), the four FE states are obtained demonstrating a specially arranged transition dipole moments (seen in Figure 2a). For the H-aggregates, the transition dipole moments of the two monomers counteract each other at the 1st and the 3rd FE states while they superpose at the 2nd and the 4th FE states, which result in the increase of absorption and emission intensities in the formers and the decrease of the ones in the latter. It is the opposite case for the J-aggregates that the absorption and emission intensi- ties of the 1st and 3rd FE states are enhanced while the ones of 2 nd and 4th FE states are reduced.

In order to figure out the influence of the vibronic coupling, we calculated the optical spectra of the model dimer as well as that of the monomer for comparison by setting $\varepsilon=3.0 \mathrm{eV}, J= \pm 50 \mathrm{meV}, \omega=1400 \mathrm{~cm}^{-1}, S=1, T=298 \mathrm{~K}$ in Figure 2. It is clearly seen that the absorption and emission spectra of the monomer both exhibit two main peaks which correspond to the 0-0 and 0-1 transition, respectively. Whereas the absorption and emission spectra of dimer show distinct feature for the different molecular packing. The H-aggregate produces the blue-shifted absorption with maximum peak assigned as 4th FE state, while the J-aggregate generates the red-shifted one with maximum peak dressed as 1st FE state. The emission spectra of the $\mathrm{H}$-aggregate is largely red shifted $(J+\hbar \omega)$ and significantly decreased in intensity, whose maximum peak is identified as the transition from 1st FE state to the ground state with $v=1$. While the emission spectra of the J-aggregate is red shifted to some extent and is hugely enhanced relative to that of the monomer, whose maximum peak is assigned as the transition from the 1st FE state to the ground state with $v=0$.

\subsubsection{Molecular parameters governing the optical spectra}

The vibronic coupling characterizes the intramolecular geometrical organization upon absorbing or emitting a phonon, which is always directly measured by the reorganization energy $(\lambda, \lambda=S \hbar \omega)$. The exciton coupling $J$ reflects the degree of the intermolecular resonant conversion, which is determined by the transition dipole moment and the intermolecular distance. Whether the intramolecular $\lambda$ or intermolecular $J$ is dominant in the absorption and emission processes can lead to different feature in absorption and emission spectra (see Figure 3). Here, we analyzed the evolution of optical spectra with $\varepsilon=3.0 \mathrm{eV}, J=$ $86.6 \mathrm{meV}$ and $\omega=1400 \mathrm{~cm}^{-1}$ by changing $\lambda$ or $S$ at $298 \mathrm{~K}$. As seen from Figure 3 , when $\lambda=J$, the exciton coupling

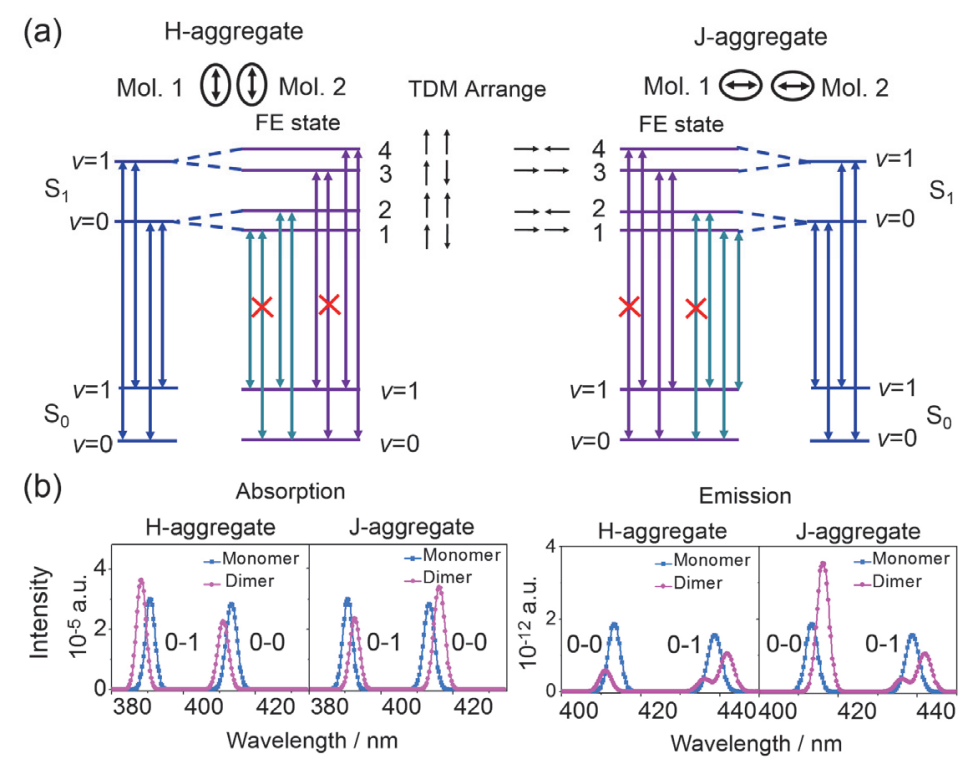

Figure 2 Vibronic coupled exciton band energy diagram (a) and the optical spectra (b, c) at $298 \mathrm{~K}$ for $\mathrm{H}$ - and $\mathrm{J}$-aggregates by setting $\varepsilon=3.0 \mathrm{eV}, J=50$ $\mathrm{meV}, \omega=1400 \mathrm{~cm}^{-1}$ and $S=1$. TDM: transition dipole moment 

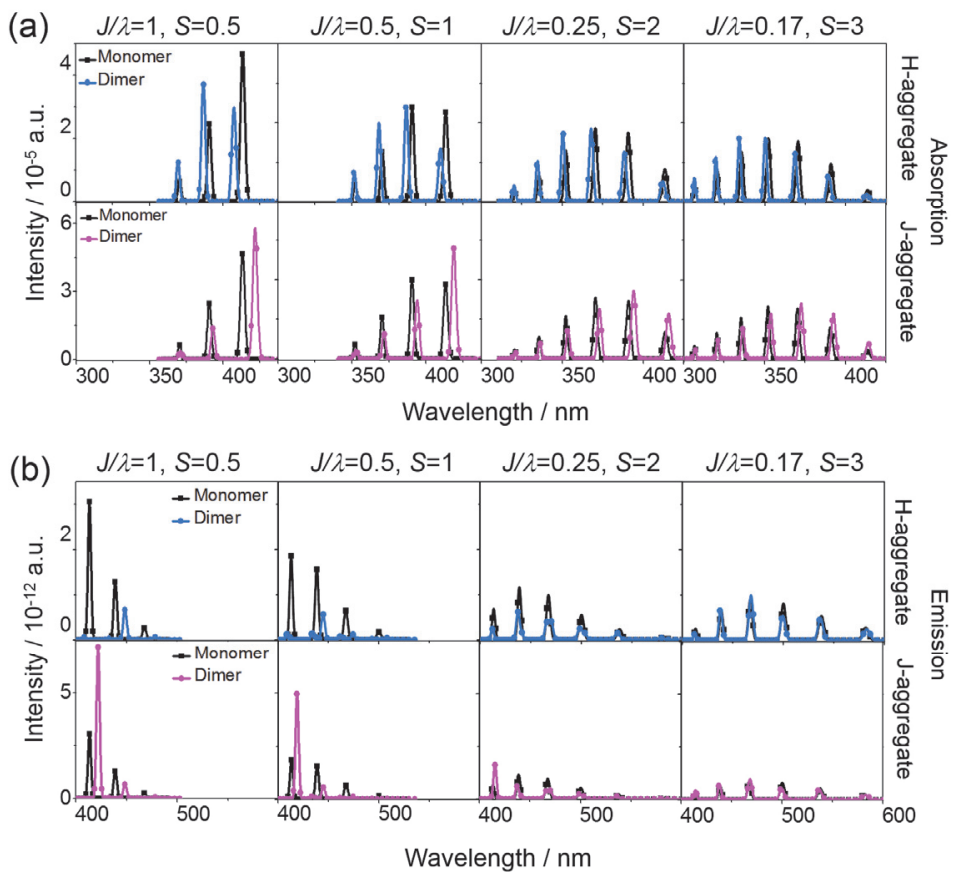

Figure 3 The dependencies of absorption (a) and emission (b) spectra on the Huang-Rhys factors at $298 \mathrm{~K}$ for $\mathrm{H}$ - and J-aggregates by setting $\varepsilon=3.0 \mathrm{eV}$, $J=86.6 \mathrm{meV}$ and $\omega=1400 \mathrm{~cm}^{-1}$

would have a large effect on the spectra and lead to remarkable spectral change relative to those of the monomer in both $\mathrm{H}$ - and J-aggregates. As the $\lambda$ increases, the change tends to be small. Then, when $\lambda$ is further increased to $J / \lambda$ $=0.17$, the spectra almost overlap with those of the monomer. It should be noted that the exciton coupling effect would be more remarkable as the temperature decreases, and a smaller critical $J / \lambda$ value is obtained for the overlap of the dimer and the monomer spectra at low temperature (See Figure S1 of SI).

\subsection{Comparison of optical spectra for Non-AIEgens and AIEgens}

To demonstrate the general behavior, we further investigated the optical spectra of a series of fluorophores, including four conventional non-AIEgens (DSB, ${ }^{[15]} 6 \mathrm{~T}^{[16]}$ Anthracene, ${ }^{[17]}$ Rubrene ${ }^{[18]}$ ) and five AIEgens (DCDPP, ${ }^{[19]}$ $\mathrm{CB}^{[15 \mathrm{a}, 20]}$ HPS, ${ }^{[15 \mathrm{a}, 21,22 \mathrm{a}, 24]}$ BFTPS, $\left.{ }^{[22]} \mathrm{BTPES}^{[22 \mathrm{a}, 23]}\right)$, see Chart 1. As known from the analysis above, the reorganization energy $(\lambda)$ and exciton coupling $(J)$ are two important competing factors in governing the spectral feature in aggregate. We first calculated the two parameters for all the studied molecular crystalline aggregates and present the results in Figure 4 (detailed data can be found in Table S1 of SI). Herein, the maximum exciton coupling value among different dimers in crystal is selected to characterize representatively the intermolecular interaction (detailed exciton coupling values of different dimers for all the studied systems can be found in Figure S2 $\sim \mathrm{S} 10$ of SI).

It is interesting to observe from Figure 4 that the ratio $J / \lambda$ for all the AIEgens is always less than 0.17 but for non-AIEgens $J / \lambda$ is always greater than 0.17 . Especially for DSB and 6T with typical aggregation caused quenching (ACQ) property, the $J / \lambda$ ratio is well above the critical value.
Figure 5, Figure 6 and Figure S11 plot the calculated crystalline optical spectra with and without exciton coupling for the non-AIEgens and AIEgens at room temperature, respectively. In the spectrum calculations, the used parameters were given in Table $\mathrm{S} 2 \sim \mathrm{S} 5$ of SI. As expected, the spectral fine structures of the non-AIEgens change apparently after the exciton coupling is taken into account, while the ones of AIEgens are almost unchanged. This fact confirms our previous observation that the influence of the exciton coupling becomes weak with the decrease of $J / \lambda$ and tends to disappear as $J / \lambda<0.17$. Among the non-AIEgens, the spectra of DSB and 6T experience the largest modification owing to very large $J / \lambda$, and the ones of anthracene and rubrene show slight excitonic effect because of small $J / \lambda$. The absorption spectra of DSB and 6T are sharply blue-shifted and their intensities are significantly enhanced, and the emissions are red-shifted with reduced intensity, which are in agreement with the typical H-type aggregation of DSB and 6T's crystals. For the AIEgens, the crystal packing is usually loose and the molecular geometry is usually flexible, leading to small $J$ and large $\lambda$ concomitantly, in another word $J / \lambda<0.17$. Thus, their spectra are independent of the exciton coupling. This justifies our previous work on the AIEgens in which the radiative and nonradiative decay rates were calculated by neglecting the exciton couplings. ${ }^{[12,15 a, 22 a, 24]}$

In order to get deep insight into the effect of exciton interaction on the optical spectrum, we took DSB and HPS as examples to dissect the origin of changes in the emission spectrum by comparing the optical spectra in gas phase with those in cluster, taking intermolecular electrostatic interaction and excitonic coupling into accounts in Figure 7 and Figure 8 where the available experimental 


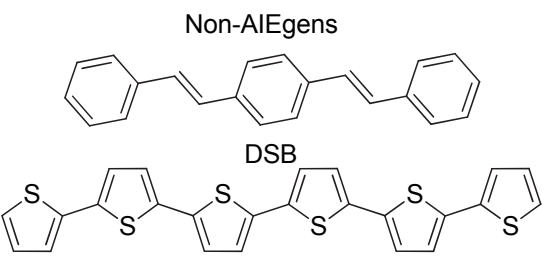

$6 \mathrm{~T}$
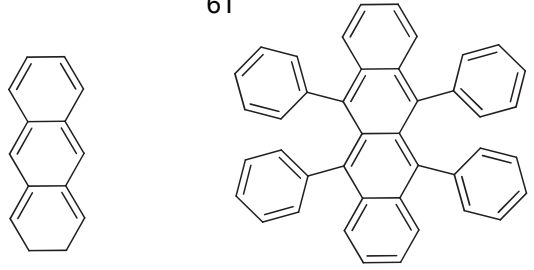

Anthracene

Rubrene

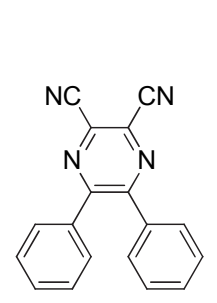

DCDPP

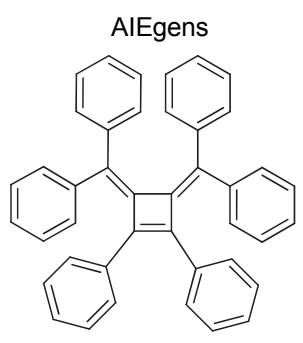

$\mathrm{CB}$

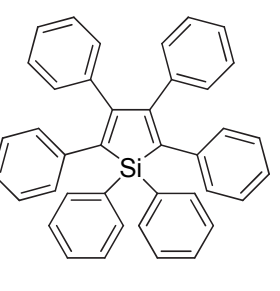

HPS
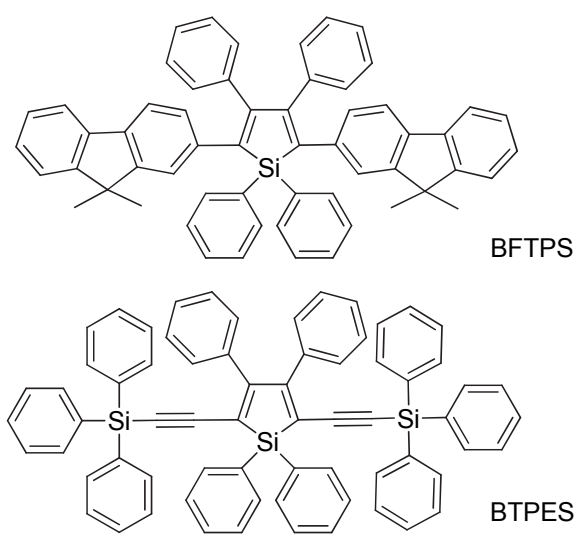

Chart 1 Molecular structures of the studied molecules

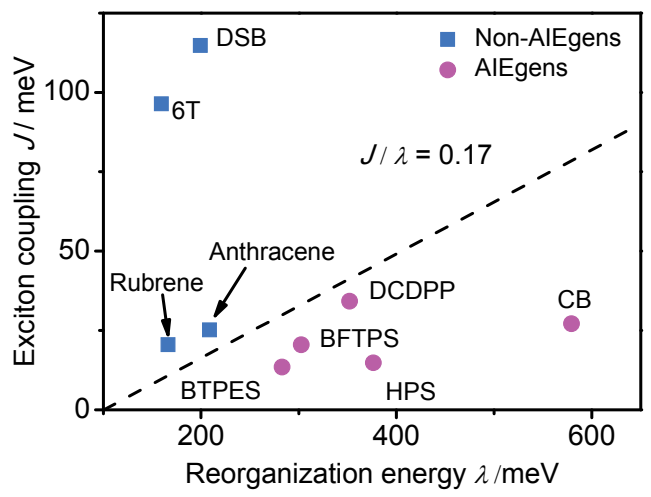

Figure 4 Calculated reorganization energies and exciton couplings of the studied molecular systems

results are also depicted. Here, the intermolecular electrostatic interaction is considered during the geometry optimizations and frequency calculations by using QM/MM
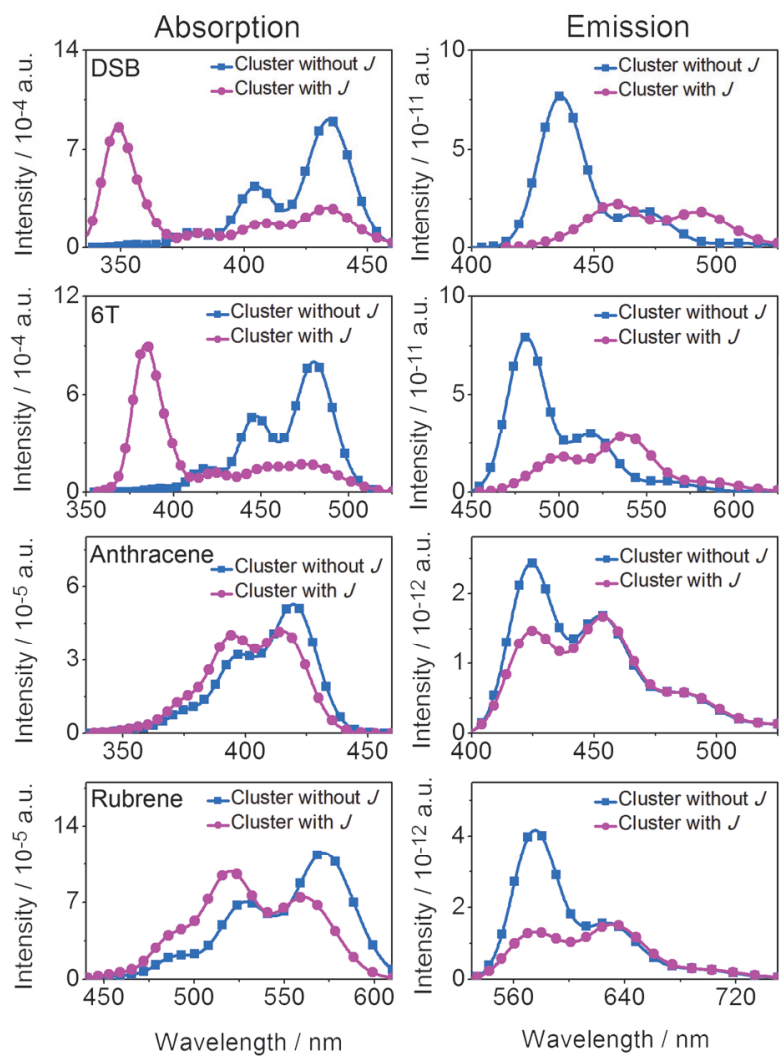

Figure 5 Optical spectra of the non-AIEgens in cluster with and without considering exciton coupling $(J)$ at $298 \mathrm{~K}$
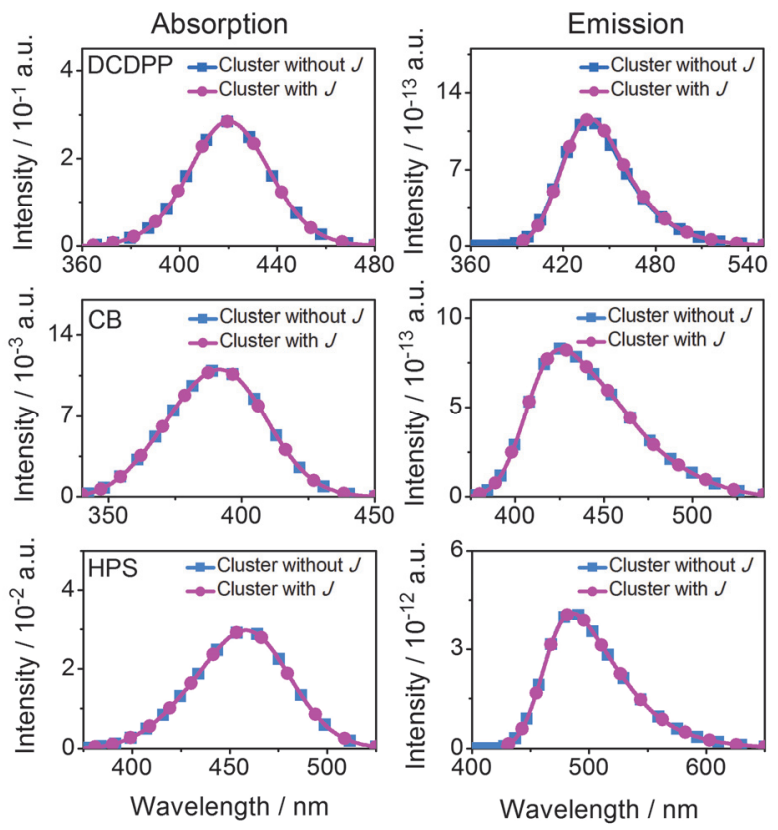

Figure 6 Optical spectra of the AIEgens (DCDPP, CB, HPS) in cluster with and without considering exciton coupling $(J)$ at $298 \mathrm{~K}$

approach. That is, the intramolecular vibronic coupling in cluster is obtained based on the electronic structure information, which includes the influence of electrostatic interaction. 


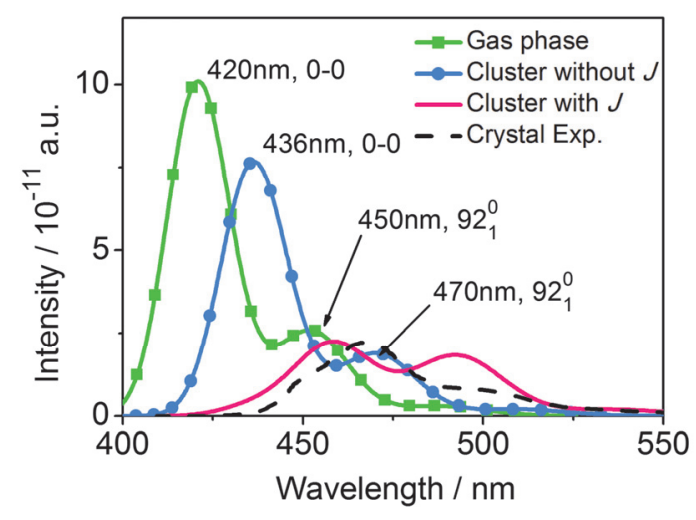

Figure 7 The calculated emission spectra of DSB in the gas phase, in a cluster with and without considering $J$ at $298 \mathrm{~K}$, as well as the experimental one in ref. $15 b^{[15 b]}$. The $92_{1}^{0}$ means the $0-1$ transition of mode 92 , $\omega_{92}=1647 \mathrm{~cm}^{-1}$ in the gas phase, $\omega_{92}=1658 \mathrm{~cm}^{-1}$ in the crystal

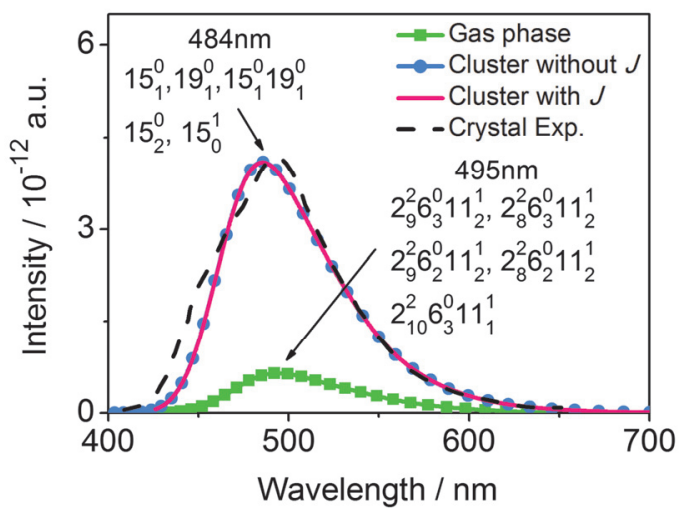

Figure 8 The calculated emission spectra of HPS in the gas phase, in a cluster with and without considering $J$ at $298 \mathrm{~K}$, as well as the experimental one in ref. $24 \mathrm{~b}^{[24 \mathrm{~b}]}$. The $15_{1}^{0}$ means the $0-1$ transition of mode $15, \omega_{2}=21 \mathrm{~cm}^{-1}, \omega_{6}=54 \mathrm{~cm}^{-1}$ and $\omega_{11}=70 \mathrm{~cm}^{-1}$ in the gas phase, $\omega_{15}=$ $137 \mathrm{~cm}^{-1}$ and $\omega_{19}=188 \mathrm{~cm}^{-1}$ in the crystal

It is obvious in Figure 7 that for DSB the emission spectra in three cases consist of two significant peaks. The two peaks are $0-0$ peak and $0-1$ peak arising from the normal mode of $c a .1650 \mathrm{~cm}^{-1}$ for the gas-phase DSB and cluster without considering $J$, respectively. The emission spectrum is red shifted from gas-phase to cluster without considering $J$ owing to the decrease of $0-0$ transition energy $\left(\Delta E_{0-0}\right)$. When taking the exciton coupling into consideration, the 0-0 peak noticeably slumps owing to the offset of the two oppositely oriented transition dipole moment. And the transitions of lower FE state mixing electronic and vibrational states become dominant, which leads to more pronounced red shift, reproducing the experimental results.

Differently from DSB, the emission spectra of HPS (in Figure 8 ) are one broad band without any fine structure. The band is assigned as mixed 2-8, 2-9, 2-10, 0-3, 0-2, 1-2, and $1-1$ transitions of low-frequency modes $\left(<100 \mathrm{~cm}^{-1}\right)$ for gas-phase HPS, and mixed 0-1, 0-2 and 1-0 transitions of low-frequency $\left(<200 \mathrm{~cm}^{-1}\right)$ for cluster without considering $J$. More strikingly, the emission spectrum is sharply enhanced in intensity and blue shifted from $495 \mathrm{~nm}$ to 484 $\mathrm{nm}$ when going from gas phase to cluster without $J$. This is easily understood that the intermolecular electrostatic interaction restricts molecular structural relaxation, namely out-of-plane twists, and decreases the vibronic coupling. ${ }^{[12 a]}$ In addition, the exciton coupling has no effect on the emission spectrum of HPS, as its $J / \lambda<0.17$. This indicates that it is enough to reproduce the experimental result by considering the intermolecular electrostatic interaction. It is learned that the exciton coupling is necessary to be considered when predicting the optical spectra of the similar planar $\pi$-conjugated molecules with large $J / \lambda$. And the intermolecular electrostatic interaction always needs to be taken into consideration for the flexible AIE systems.

\section{Conclusions}

In this work, we systematically investigated the role of intramolecular vibronic coupling and intermolecular interaction in determining the optical spectrum features. Starting from vibronic-coupled exciton models, we reveal the role of the vibronic coupling in the fine structure of the optical spectrum. Then we examined the competitive relationship of the intramolecular vibronic coupling $(\lambda)$ and the intermolecular exciton coupling $(J)$ to dominate the crystalline state spectral characters. It is found that the impact of the exciton coupling on the spectra decreases with the ratio of $J / \lambda$, and goes to null when $J / \lambda<0.17$ at room temperature.

Furthermore, we compare the crystalline optical spectra of a series of non-AIEgens with AIEgens at room temperature. It is found that the spectra of the non-AIEgens are strongly dependent on the intermolecular exciton coupling but for AIEgens the ratio $J / \lambda$ is always less than 0.17 , leading to the independence of optical spectra on the exciton coupling. The spectra of typical non-AIEgens DSB and $6 \mathrm{~T}$ undergo remarkable modification from the gas phase to aggregate owing to their very large $J / \lambda$, and for anthracene and rubrene, the modification is small for the small $J / \lambda$ value close to 0.17 . But for AIEgens, the optical spectra are remarkably insensitive to the exciton coupling and the intramolecular vibronic coupling with intermolecular electrostatic interaction dominate the photophysical processes. These results help to understand the differences between AIEgens and non-AIEgens.

\section{Methodology}

\subsection{Vibronic coupled Frenkel Exciton Approach}

In the organic molecule aggregates, the Frenkel exciton (FE) model is usually adopted to describe the exciton coupling ${ }^{[8,9]}$. The basis function of the aggregate (containing $p$ monomers) at the excited state can be expressed as: $|e, n\rangle=|g(1), \cdots, g(n-1), e(n), g(n+1), \cdots, g(p)\rangle$, and the ground state is $|g\rangle=|g(1), g(2), \cdots, g(p)\rangle$. Hamiltonian matrix for the aggregate at the excited state reads,

$$
\boldsymbol{H}_{e}=\left(\begin{array}{ccccc}
H_{1} & J_{12} & \cdots & J_{1, p-1} & J_{1, p} \\
J_{21} & H_{2} & \cdots & J_{2, p-1} & J_{2, p} \\
\cdots & \cdots & \cdots & \cdots & \cdots \\
J_{p-1,1} & J_{p-1,2} & \cdots & H_{p-1} & J_{p-1, p} \\
J_{p, 1} & J_{p, 2} & \cdots & J_{p, p-1} & H_{p}
\end{array}\right)
$$


with

$$
\begin{gathered}
H_{n}=\langle e, n|\hat{H}| e, n\rangle=\langle e(n)|\hat{H}| e(n)\rangle+\sum_{m \neq n}^{p}\langle g(m)|\hat{H}| g(m)\rangle \\
J_{m, n}=\langle e, m|\hat{H}| e, n\rangle
\end{gathered}
$$

Assuming the harmonic oscillator and one-particle approximation (in which the vibration states of the excited molecule are active while the ones of other molecules are frozen ${ }^{[9]}$ ), the elements in Eq. (4) are recast as,

$$
\begin{aligned}
H_{n, \mathbf{v}} & =\left\langle\Psi_{n, \mathbf{v}}^{e}|\hat{H}| \Psi_{n, \mathbf{v}}^{e}\right\rangle-\left\langle\Psi_{n, \mathbf{0}}^{g}|\hat{H}| \Psi_{n, \mathbf{0}}^{g}\right\rangle \\
& =\varepsilon_{n}^{\mathrm{ad}}+\sum_{k} \sum_{v_{k}}\left(\frac{1}{2}+v_{k}\right) \hbar \omega_{n k}^{e} \\
J_{m, n, \mathrm{v}_{m}, \mathrm{v}_{n}} & =\left\langle\Psi_{m, \mathrm{v}_{m}}^{e} \Psi_{n, \mathbf{0}}^{g}|\hat{H}| \Psi_{m, \mathbf{0}}^{g} \Psi_{n, \mathrm{v}_{n}}^{e}\right\rangle \\
& =\left\langle\Phi_{m}^{e} \Phi_{n}^{g}|\hat{H}| \Phi_{m}^{g} \Phi_{n}^{e}\right\rangle\left\langle\Theta_{m, \mathrm{v}_{m}}^{e} \Theta_{n, \mathbf{0}}^{g} \mid \Theta_{m, \mathbf{0}}^{g} \Theta_{n, \mathrm{v}_{n}}^{e}\right\rangle \\
& =\left\langle\Phi_{m}^{e} \Phi_{n}^{g}|\hat{H}| \Phi_{m}^{g} \Phi_{n}^{e}\right\rangle \prod_{i=m, n} \prod_{k} f_{k, v_{i k}-0}^{i}
\end{aligned}
$$

Here, $m / n$ is the index of the excited monomer; $\boldsymbol{v}_{n} \equiv\left(v_{1}, \cdots, v_{k-1}, v_{k}, \cdots, v_{3 n-6}\right), v_{k}$ is the vibrational quantum number of the $k^{\text {th }}$ normal mode; $\varepsilon_{n}^{\text {ad }}$ is the adiabatic excitation energy of the $n^{\text {th }}$ molecule; $\omega_{n k}^{e}$ is the frequency of the $n^{\text {th }}$ molecule in the excited state; $f_{k, v_{i k}-0}^{i}$ is the Franck-Condon (FC) integral from ground-state 0 to $v$ excited-state for the $k^{\text {th }}$ normal mode of the $i^{\text {th }}$ molecule.

Only the Coulomb potential is considered in $\left\langle\Phi_{m}^{e} \Phi_{n}^{g}|\hat{H}| \Phi_{m}^{g} \Phi_{n}^{e}\right\rangle^{[25]}$ :

$\left\langle\Phi_{m}^{e} \Phi_{n}^{g}|\hat{H}| \Phi_{m}^{g} \Phi_{n}^{e}\right\rangle \approx J_{\text {coul }}=\int \mathrm{d} \vec{r} \int \mathrm{d} \vec{r}^{\prime} \rho_{m}^{*}(\vec{r}) \frac{1}{\left|r-r^{\prime}\right|} \rho_{n}\left(\vec{r}^{\prime}\right)$

Here $\rho_{m}(\vec{r})$ and $\rho_{n}(\vec{r})$ are the excited-state transition densities for the $m^{\text {th }}$ and $n^{\text {th }}$ molecules, respectively.

By diagonalizing the matrix Eq. (4), we can obtain the eigenvalue (eigenenergy) and eigenvector (eigenstate) of the excited states. Then, we can calculate the absorption and emission spectra based on Eq. (8) and Eq. $(9)^{[14]}$ :

$$
\begin{aligned}
\sigma_{\text {abs }}(\omega) & =\frac{4 \pi^{2} \omega}{3 \hbar c}|\vec{\mu}|^{2}|F C|^{2} \delta(\Delta \varepsilon-\omega) \\
\sigma_{\text {emi }}(\omega) & =\frac{4 \pi^{2} \omega^{3}}{3 \hbar c^{3}}|\vec{\mu}|^{2}|F C|^{2} \delta(\Delta \varepsilon-\omega)
\end{aligned}
$$

Here, $\vec{\mu}$ is the monomer transition dipole moment; $\Delta \varepsilon$ is the energy difference between the excited state and ground state of the aggregate; FC integral can be obtained from the eigenfunction of the aggregate's excited and ground states.

\subsection{Computational Details}

The quantum calculations of all the studied compounds are performed out by using QM/MM approach, including geometrical optimization, electronic structure, vibration frequency, transition property and so on. The QM/MM models are set up by cutting out a cluster from the X-ray crystal structure and prescribing the central molecule as QM part and its surrounding ones as MM part. Taking distyrylbenzene (DSB) as an example, the QM/MM cluster (Chart 2) size is $5 \times 5 \times 3$ and the thirdly nearest molecules are taken into account in the compact herringbone directions (total 105 molecules). (TD) B3LYP ${ }^{[26]}$ and 6-31G(d) were used for QM calculation. General Amber force field (GAFF) was adopted to describe the MM molecules. ${ }^{[27]}$ The QM/MM calculation was accomplished by using ChemShell 3.5 package, ${ }^{[28]}$ which interfaces the Turbomole 6.5 program $^{[29]}$ for QM and DL_POLY program ${ }^{[30]}$ for MM. It should be noted that the QM molecule was active while the MM molecules were kept frozen and the electrostatic embedding scheme with QM polarization was adopted. In addition, the exciton couplings $(\mathrm{J})$ were calculated at the level of CAM-B3LYP ${ }^{[31]} / 6-31 \mathrm{G}(\mathrm{d})$ by using our home-built MOMAP program ${ }^{[32]}$ coupling with the NWchem 6.3 program. ${ }^{[33]}$

A Frenkel exciton computational model consists of a central monomer and its most adjacent molecules in the crystal $(8 \sim 9$ molecules, shown in Figure $\mathrm{S} 2 \sim \mathrm{S} 10$ of $\mathrm{SI})$. The optical spectra were evaluated by employing the vibronic coupled Frenkel exciton theory in MOMAP program. Herein, the Lorentzian broadening was applied with a constant $\gamma=1100 \mathrm{~cm}^{-1}$ for non-AIEgens, ${ }^{[34]}$ and 2000 $\mathrm{cm}^{-1}$ for AIEgens to reproduce the spectrum with fully vibrational states obtained by the vibration correlation function approach at room temperature. ${ }^{[24]}$ To balance the computational cost and accuracy, the important normal modes were selected (see details in Table S2 $\mathrm{S} 5$ ), and its vibrational states were cut off when the FC integral $f_{v_{k}-0}$ less than $1 \%$ of the maximum.

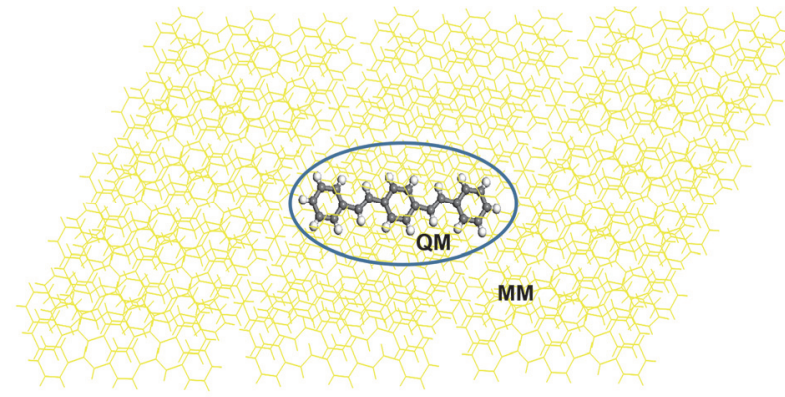

Chart 2 QM/MM computational model of DSB with one central QM molecule and surrounding $104 \mathrm{MM}$ molecules

\section{Supporting Information}

Diagonalization of model exciton Hamiltonian in Eq. (3), the reorganization energy and exciton coupling of the selected fluorophores; The temperature dependencies of optical spectra in the $\mathrm{H}$ - and J-aggregates; the Frenkel exciton computational model cutting off from crystal and the relative exciton coupling values; optical spectra of the AIEgens (BFTPS and BTPES) in cluster with and without exciton coupling at $298 \mathrm{~K}$; the selected normal modes and their quantum number of the Non-AIEgens and AIEgens. 


\section{References}

[1] (a) Tang, C. W.; VanSlyke, S. A. Appl. Phys. Lett. 1987, 51, 913; (b) Burroughes, J. H.; Bradley, D. D. C.; Brown, A. R.; Marks, R. N.; Mackay, K.; Friend, R. H.; Burns, P. L.; Holmes, A. B. Nature 1990, 347, 539; (c) Malissa, H.; Kavand, M.; Waters, D. P.; van Schooten, K. J.; Burn, P. L.; Vardeny, Z. V.; Saam, B.; Lupton, J. M.; Boehme, C. Science 2014, 345, 1487; (d) Lee, J.; Chen, H.-F.; Batagoda, T.; Coburn, C.; Djurovich, P. I.; Thompson, M. E.; Forrest, S. R. Nat. Mater. 2016, 15, 92.

[2] (a) Schäfer, F. P.; Schmidt, W.; Volze, J. Appl. Phys. Lett. 1966, 9, 306; (b) Morales-Vidal, M.; Boj, P. G.; Villalvilla, J. M.; Quintana, J. A.; Yan, Q.; Lin, N.-T.; Zhu, X.; Ruangsupapichat, N.; Casado, J.; Tsuji, H.; Nakamura, E.; Diaz-Garcia, M. A. Nat. Commun. 2015, 6, 8458 .

[3] (a) Horowitz, G. Adv. Mater. 1998, 10, 365; (b) Liu, J.; Zhang, H.; Dong, H.; Meng, L.; Jiang, L.; Jiang, L.; Wang, Y.; Yu, J.; Sun, Y.; Hu, W.; Heeger, A. J. Nat. Commun. 2015, 6, 10032.

[4] (a) Gaylord, B. S.; Heeger, A. J.; Bazan, G. C. PNAS 2002, 99, 10954; (b) Rana, S.; Elci, S. G.; Mout, R.; Singla, A. K.; Yazdani, M.; Bender, M.; Bajaj, A.; Saha, K.; Bunz, U. H. F.; Jirik, F. R.; Rotello, V. M. J. Am. Chem. Soc. 2016, 138, 4522.

[5] (a) Yu, G.; Gao, J.; Hummelen, J. C.; Wudl, F.; Heeger, A. J. Science 1995, 270, 1789; (b) Page, Z. A.; Liu, Y.; Duzhko, V. V.; Russell, T. P.; Emrick, T. Science 2014, 346, 441; (c) Holliday, S.; Ashraf, R. S.; Wadsworth, A.; Baran, D.; Yousaf, S. A.; Nielsen, C. B.; Tan, C. H.; Dimitrov, S. D.; Shang, Z. R.; Gasparini, N.; Alamoudi, M.; Laquai, F.; Brabec, C. J.; Salleo, A.; Durrant, J. R.; McCulloch, I. Nat. Commun. 2016, 7, 11; (d) Rong, Y.; Mei, A.; Liu, L.; Li, X.; Han, H. Acta Chim. Sinica 2015, 73, 237. (荣耀光, 梅安意, 刘林峰, 李雄, 韩宏伟，化学学报, 2015, 73, 237.) (e) Fu, Y.-T.; Yi, Y.; Coropceanu, V.; Risko, C.; Aziz, S. G.; Brédas, J.-L. Sci. China Chem. 2014, $57,1330$.

[6] Mei, J.; Leung, N. L. C.; Kwok, R. T. K.; Lam, J. W. Y.; Tang, B. Z. Chem. Rev. 2015, 115, 11718

[7] Valeur, B.; Berberan-Santos, M. N. Molecular Fluorescence, Wiley-VCH, Weinheim, 2012, pp. 141 179 .

[8] (a) Kasha, M. Radiat. Res. 1963, 20, 55; (b) Kasha, M.; Rawls, H.; El-Bayoumi, M. A. Pure Appl. Chem. 1965, 11, 371 .

[9] (a) Spano, F. C. Acc. Chem. Res. 2010, 43, 429; (b) Spano, F. C. J. Chem. Phys. 2003, 118, 981; (c) Spano, F. C. Phys. Rev. B 2005, 71, 094110; (d) Spano, F. C. Annu. Rev. Phys. Chem. 2006, 57, 217.

[10] (a) Wykes, M.; Parambil, R.; Beljonne, D.; Gierschner, J. J. Chem. Phys. 2015, 143, 114116; (b) Gierschner, J.; Ehni, M.; Egelhaaf, H.-J.; Milián Medina, B.; Beljonne, D.; Benmansour, H.; Bazan, G. C. J. Chem. Phys. 2005, 123, 144914.

[11] Gao, F.; Liang, W.; Zhao, Y. Sci. China Chem. 2010, 53, 297.

[12] (a) Wu, Q.; Zhang, T.; Peng, Q.; Wang, D.; Shuai, Z. Phys. Chem. Chem. Phys. 2014, 16, 5545. (b) Wu, Q.; Peng, Q.; Zhang, T.; Shuai, Z. Sci. China Chem. 2013, 43, 1078.

[13] Kasha, M. Discuss. Faraday Soc. 1950, 9, 14.

[14] Niu, Y.; Peng, Q.; Deng, C.; Gao, X.; Shuai, Z. J. Phys. Chem. A 2010, 114, 7817;

[15] (a) Zhang, T.; Peng, Q.; Quan, C.; Nie, H.; Niu, Y.; Xie, Y.; Zhao, Z.; Tang, B. Z.; Shuai, Z. Chem. Sci. 2016, 7, 5573; (b) Wu, C. C.; Korovyanko, O. J.; Delong, M. C.; Vardeny, Z. V.; Ferraris, J. P. Synth. Met. 2003, 139, 735 .

[16] (a) Yassar, A.; Horowitz, G.; Valat, P.; Wintgens, V.; Hmyene, M.; Deloffre, F.; Srivastava, P.; Lang, P.; Garnier, F. J. Phys. Chem. 1995, 99, 9155; (b) Stradomska, A.; Petelenz, P. J. Chem. Phys.
2009, 130, 094705.

[17] (a) Mason, R. Acta Crystallogr. 1964, 17, 547; (b) Pope, M.; Kallmann, H. P.; Magnante, P. J. Chem. Phys. 1963, 38, 2042; (c) Li, H.; Duan, L.; Zhang, D.; Dong, G.; Wang, L.; Qiu, Y. Sci. China Ser. B: Chem. 2009, 52, 181 .

[18] (a) Gao, F.; Liang, W. Z.; Zhao, Y. J. Phys. Chem. A 2009, 113 , 12847; (b) Mitrofanov, O.; Kloc, C.; Siegrist, T.; Lang, D. V.; So, W.-Y.; Ramirez, A. P. Appl. Phys. Lett. 2007, 91, 212106.

[19] (a) Wu, Q.; Deng, C.; Peng, Q.; Niu, Y.; Shuai, Z. J. Comput. Chem. 2012, 33, 1862; (b) Qin, A.; Lam, J. W. Y.; Mahtab, F.; Jim, C. K W.; Tang, L.; Sun, J.; Sung, H. H. Y.; Williams, I. D.; Tang, B. Z. Appl. Phys. Lett. 2009, 94, 253308.

[20] Dong, Y.; Lam, J. W. Y.; Qin, A.; Sun, J.; Liu, J.; Li, Z.; Sun, J.; Sung, H. H. Y.; Williams, I. D.; Kwok, H. S.; Tang, B. Z. Chem. Commun. 2007, 31, 3255.

[21] Chen, J.; Law, C. C. W.; Lam, J. W. Y.; Dong, Y.; Lo, S. M. F.; Williams, I. D.; Zhu, D.; Tang, B. Z. Chem. Mater. 2003, 15, 1535.

[22] (a) Xie, Y.; Zhang, T.; Li, Z.; Peng, Q.; Yi, Y.; Shuai, Z. Chem. Asian J. 2015, 10, 2154; (b) Zhan, X.; Haldi, A.; Risko, C.; Chan, C. K.; Zhao, W.; Timofeeva, T. V.; Korlyukov, A.; Antipin, M. Y.; Montgomery, S.; Thompson, E.; An, Z.; Domercq, B.; Barlow, S.; Kahn, A.; Kippelen, B.; Bredas, J.-L.; Marder, S. R. J. Mater. Chem. 2008 $18,3157$.

[23] Zhao, Z.; Liu, D.; Mahtab, F.; Xin, L.; Shen, Z.; Yu, Y.; Chan, C. Y. K.; Lu, P.; Lam, J. W. Y.; Sung, H. H. Y.; Williams, I. D.; Yang, B.; Ma, Y.; Tang, B. Z. Chem.-Eur. J. 2011, 17, 5998.

[24] (a) Zhang, T.; Jiang, Y.; Niu, Y.; Wang, D.; Peng, Q.; Shuai, Z. J. Phys. Chem. A 2014, 118, 9094. (b) Zhan, X. W.; Risko, C.; Amy, F.; Chan, C.; Zhao, W.; Barlow, S.; Kahn, A.; Bredas, J.-L.; Marder, S. R. J. Am. Chem. Soc. 2005, 127, 9021.

[25] Hsu, C.-P.; You, Z.-Q.; Chen, H.-C. J. Phys. Chem. C 2008, 112, 1204.

[26] (a) Becke, A. D. J. Chem. Phys. 1993, 98, 5648; (b) Lee, C.; Yang, W.; Parr, R. G. Phys. Rev. B 1988, 37, 785.

[27] Wang, J.; Wolf, R. M.; Caldwell, J. W.; Kollman, P. A.; Case, D. A. J. Comput. Chem. 2004, 25, 1157.

[28] Sherwood, P.; de Vries, A. H.; Guest, M. F.; Schreckenbach, G.; Catlow, C. R. A.; French, S. A.; Sokol, A. A.; Bromley, S. T.; Thiel, W.; Turner, A. J.; Billeter, S.; Terstegen, F.; Thiel, S.; Kendrick, J.; Rogers, S. C.; Casci, J.; Watson, M.; King, F.; Karlsen, E.; Sjøvoll, M.; Fahmi, A.; Schäfer, A.; Lennartz, C. J. Mol. Struct. THEOCHEM 2003, 632, 1.

[29] (a) TURBOMOLE V6.5 2013, University of Karlsruhe and of the Forschungszentrum Karlsruhe GmbH, 1989-2007; TURBOLE GmbH, since 2007 (accessed May 23, 2013); (b) Ahlrichs, R.; Bär, M.; Häser, M.; Horn, H.; Kölmel, C. Chem. Phys. Lett. 1989, 162, 165.

[30] Smith, W.; Forester, T. R. J. Mol. Graphics 1996, 14, 136.

[31] Yanai, T.; Tew, D. P.; Handy, N. C. Chem. Phys. Lett. 2004, 393, 51.

[32] Shuai, Z.; Peng, Q.; Niu, Y.; Geng, H.; MOMAP, Revision 0.3.001 ed.; MOMAP: a free and open-source molecular materials property prediction package; avaliable online : http://www.shuaigroup.net, Beijing, China, 2016.

[33] Valiev, M.; Bylaska, E. J.; Govind, N.; Kowalski, K.; Straatsma, T. P.; Van Dam, H. J. J.; Wang, D.; Nieplocha, J.; Apra, E.; Windus, T. L.; de Jong, W. A. Comput. Phys. Commun. 2010, 181, 1477.

[34] Gierschner, J.; Mack, H. G.; Egelhaaf, H. J.; Schweizer, S.; Doser, B.; Oelkrug, D. Synth. Met. 2003, 138, 311.

(Cheng, B.) 